\title{
Multilocular Thymic Cyst Resection From Pleural Cavity by Thorascopy
}

\author{
Miklos Boros ${ }^{\mathrm{a}, \mathrm{c}}$, Szabolcs Molnar ${ }^{\mathrm{a}}$, Tamas Jozsa ${ }^{\mathrm{b}}$
}

\begin{abstract}
Thoracic cystic lesions are rare and mostly asymptomatic therefore they can cause differential diagnostic problems in childhood. In our case a 2-year-old boy was treated with pleuropneumonia. After antibiotic treatment there was no regression on CXR, CT showed a huge paracardial cystic lesion in left pleural cavity. Complete resection was performed by thoracoscopy, postoperative histological diagnosis was benign multilocular thymic cyst. Thymic cysts are rare in childhood, therefore it can cause diagnostic problems, mostly when signs and complains are suggest more common disease.
\end{abstract}

Keywords: Thymic cyst; Benign; Pleural cavity; Thoracoscopy

\section{Introduction}

Thymic cysts are rare lesions of the mediastinum, congenital thymic cysts accounting for less than $1 \%$ of anterosuperior mediastinal masses with no malignant potential [1]. With regard to the embryology of the thymus, the presence of a congenital thymic cyst outside the mediastinum is very rare, and thymic cysts located solely in the pleural space have been described in only one case report [2]. In our case congenital thymic cyst did not show any connection with the thymus and thoracoscopy was used for complete resection in childhood.

\footnotetext{
Manuscript accepted for publication January 22, 2013

${ }^{a}$ Division of Pediatric Surgery, Department of Pediatrics, Medical- and Health Science Center, University of Debrecen, Debrecen, Hungary

${ }^{b}$ Department of Pediatric Surgery, Karolinska Institute, Stockholm, Sweden

${ }^{\mathrm{c} C}$ Corresponding author: Miklos Boros, H-4032, Debrecen, Nagyerdei str.62/A, Hungary. Email: borosmiklosdr@hotmail.com
}

doi: http://dx.doi.org/10.4021/jmc1081w

\section{Case Report}

A 2-year-old boy underwent emergency admission with severe respiratory symptoms, coughing and dyspnea. For 2 days before admission, he had been feverish and had displayed a poor appetite. His medical history was unremarkable and no previous cardiac or pulmonary events were reported. The chest X-ray showed a paracardial mass and a small amount of free fluid in the left thoracic cavity. The laboratory tests were negative (normal WBC count and CRP). Ultrasonography demonstrated fluid in the lower part of the left thoracic cavity. After hospitalization antibiotic therapy was started. On the third day after admission, there was no fever, but the chest X-ray revealed an unchanged radiological status. A thoracic CT scan confirmed a paracardial cystic lesion without free fluid and compression atelectatics of the left lower lobe, assumed to be a pericardial cyst (Fig. 1). The CT did not indicate any connection with the anterior mediastinum. The antibiotic therapy was terminated and leftsided thoracoscopy was performed under selective intubation. A huge multilocular cystic lesion and a little free fluid in the left thoracic cavity were found during the operation.

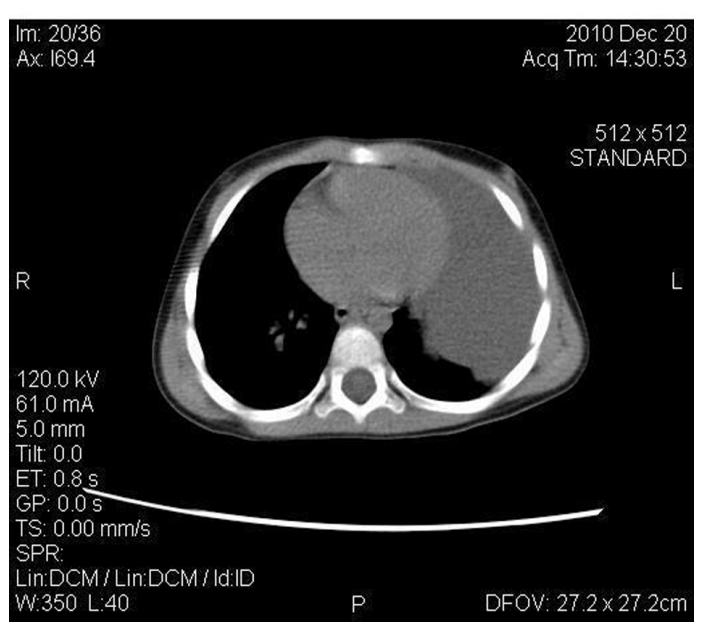

Figure 1. A huge cyst in the left pleural cavity without any connection with the retrosternal region. 


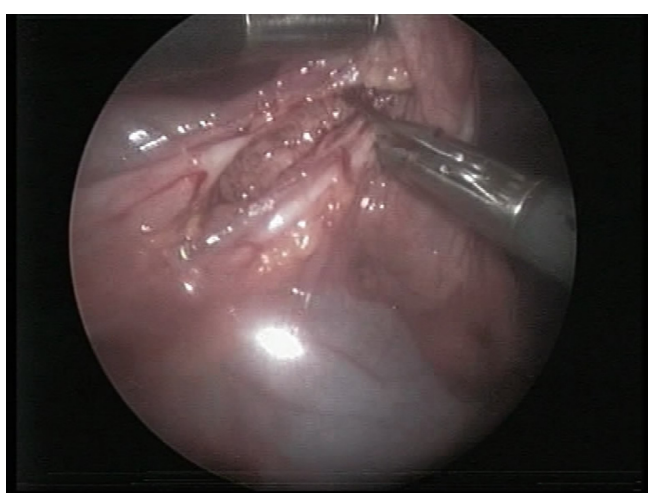

Figure 2. Cystic wall was involved by phrenic nerve.

The surface of the cyst was covered by pericardial fat tissue. The mass was attached to the pericardium, and there was no connection with the anterior-upper mediastinum, thymus or lung. After multiple fenestrations, we removed $350 \mathrm{~mL}$ of serous fluid and most of the cystic wall which had a wide connection with the pericardium and involved pericardial fat tissue and phrenic nerve (Fig. 2). We could not find a layer that could easily be prepared and large-scale resection of the pericardium would have necessitated replacement. Accordingly the internal layer of the cyst was fully resected macroscopically after dissection of the nerve, only a small part of the external layer being left on the pericardium. A $14 \mathrm{Ch}$ thoracic drain was inserted for 24 hours. The patient was discharged on day postoperative 3 in a good condition. The histologic diagnosis was a benign multilocular thymic cyst within the Hassal bodies in the cystic wall. The wall of the cyst exhibited varying thickness and contained scattered nests of thymic tissue including small lymphocytes and epithelial cells. There were no complications and no sign of recurrence during the 18-month follow-up.

\section{Discussion}

Cystic lesions of the mediastinum and thoracic cavity, which are mostly benign masses, comprise a small but important diagnostic group, constituting 12 to $18 \%$ of all primary mediastinal lesions. The classification of cysts, based on their etiology, distinguishes bronchogenic, esophageal duplication cysts, pericardial cysts, thymic cysts, and other miscellaneous cysts [3-5]. The histogenesis of ectopic thymic tissue is still unclear, the most common theory is an embryological displacement. The neck, thyroid gland, lung, middle or posterior mediastinum, pericardium and pleura have been reported as ectopic sites $[2,3,4,6]$.

Most thymic cysts can be explained as sequestered remnants of the thymopharyngeal duct system. The thymus is derived from the ventral part of the third pharyngeal pair of pharyngeal pouches. During the embryological descent from the lateral neck to the manubrium sterni, remnants of the pharyngeal system may persist and lead to the formation of congenital thymic cysts. This theory is supported by the relatively frequent observation of endocrine (mostly parathyroid) gland inclusions within the cysts [2]. Despite this, a congenital thymic cyst outside the mediastinum is extremely rare, and multilocular thymic cysts located exclusively in the pleural space have not been described. Congenital thymic cysts are usually unilocular with thin walls, and are derivatives of embryologic thymic tissue. The acquired type is often multilocular as a result of an inflammatory or neoplastic process $[7,8]$. The multilocular thymic cyst is less common than the congenital unilocular type and is believed to arise from thymic parenchymal inflammation causing cystic dilation. Multilocular cysts have been reported in association with a variety of neoplastic, autoimmune and infectious conditions [9]. Graeber et al [6] divided cystic lesions of the thymus into three major categories: congenital, neoplastic and degenerative, the latter involving two mechanisms of pathogenesis: cystic degeneration of thymomas and cystic masses. The local disruption of thymic tissue can induce the formation or growth of cysts. The reported prevalence of cysts in all mediastinal tumors of the adult population is somewhat higher than that in the pediatric population ( $14.1 \%$ vs $7.7 \%$, $\mathrm{P}<0.05)$ [4].

The diagnostic evaluation begins with the chest X-ray which identifies $89.5 \%$ of all mediastinal cysts [3]. Small subcarinal or hilar bronchogenic cysts and small thymic cysts have been revealed by chest CT. Barium swallow studies and/or esophagoscopy are performed mainly in case of suspected esophageal and bronchogenic cysts. Most of these cysts are asymptomatic, but about $6 \%$ may cause dysphagia, dyspnea, neck pain, stridor or rhonchus. They may cause complaints because of a compression closed structure, haemorrhage is a rare complication $[3,10]$. Operative indications include a cyst measuring more than $5 \mathrm{~cm}$, one that causes complaints, a multilocular cyst, diagnostic problems and the wide wall of a cyst. Most cystic lesions in the mediastinum and thoracic cavity can cause differential diagnostic problems, as in our case.

Surgical excision can be performed via an open procedure or a thoracoscopic approach; video-assisted thoracoscopy has recently become more popular in consequence of its excellent transpleural approach to the mediastinum, providing safe removal of the mass [11]. In our case, the intraoperative diagnosis was a benign disease, including such possibilities as a mediastinal cyst, a pericardial cyst or a pleural cyst, but excluding a thymic cyst, although the definitive diagnosis was possible only on histological analysis. In all cases involving a cystic lesion in the mediastinum or thoracic cavity, an effort must be made to resect it completely or, as in our case, when it is closely connected with other organs, remove the internal layer [12]. We have not found in the literature any definitive protocol for the opera- 
tive treatment of this huge, benign multilocular cystic lesion in thoracic cavity, but limited resection of the external part with total removal of the internal layer appears to be a good method for resection. It can be performed thoracoscopy and the late result is very good.

In conclusion, the intrathoracal congenital thymic cyst is a distinct clinical entity, which should be considered when the radiological findings demonstrate a rounded and welldefined paracardial mass. Thoracoscopic removal of a thymic cyst is an effective and feasible alternative in surgical treatment.

\section{Acknowledgement}

The authors thank to Dr.David Durham for critical reading of the manuscript and for linguistic corrections.

\section{Declaration}

None of the authors have anything to disclose. I am disclosing all relevant financial relationships with any commercial interests that pertain to this article.

\section{References}

1. Sameh IS, Ismaeil MF, Nasser MA, Awadalla MM. Huge cervico-thoracic thymic cyst. Interact Cardiovasc Thorac Surg. 2003;2(3):339-340.

2. Bruno VD, Mariscalco G, Franzi F, Miceli A, Piffaretti G, Sala A. Ectopic congenital thymic cyst in the right pleural cavity. Asian Cardiovasc Thorac Ann.
2010;18(5):486-488.

3. Takeda S, Miyoshi S, Minami M, Ohta M, Masaoka A, Matsuda H. Clinical spectrum of mediastinal cysts. Chest. 2003;124(1):125-132.

4. Oldham HN, Jr. Mediastinal tumors and cysts. Ann Thorac Surg. 1971;11(3):246-275.

5. Suster S, Rosai J. Multilocular thymic cyst: an acquired reactive process. Study of 18 cases. Am J Surg Pathol. 1991;15(4):388-398.

6. Graeber GM, Thompson LD, Cohen DJ, Ronnigen LD, Jaffin J, Zajtchuk R. Cystic lesion of the thymus. An occasionally malignant cervical and/or anterior mediastinal mass. J Thorac Cardiovasc Surg. 1984;87(2):295300.

7. Guba AM, Jr., Adam AE, Jaques DA, Chambers RG. Cervical presentation of thymic cysts. Am J Surg. 1978;136(4):430-436.

8. Constantacos C, Lawson NM, Votanopoulos KI, Olutoye O, Eldin KW, Feigin RD. Giant thymic cyst in left lower hemithorax of a healthy teenaged athlete. J Thorac Cardiovasc Surg. 2007;134(5):1373-1374.

9. Kasirye Y, Talsness S, Walters MP, Douglas-Jones JW, Resnick JM, Mazza JJ, Yale SH. Multilocular thymic cyst with epithelioid granulomata of unknown etiology: a radiologic and histopathologic correlation. Ann Diagn Pathol. 2012;16(1):38-42.

10. Duwe BV, Sterman DH, Musani AI. Tumors of the mediastinum. Chest. 2005;128(4):2893-2909.

11. Lachanas E, Konofaos P, Birba G, Tomos P. A rupture of a huge thymic cyst into the pleural cavity: A case report. Respir Med. 2006;100(10):1858-1860.

12. Gossot D, Izquierdo RR, Girard P, Stern JB, Magdeleinat $\mathrm{P}$. Thoracoscopic resection of bulky intrathoracic benign lesions. Eur J Cardiothorac Surg. 2007;32(6):848-851. 